ด

columns with other interested bodies where collaboration can be mutually beneficial. Links have already been formed with the National Mental Health Partnership of Chief Executives, NIMHE, British Association of Medical Managers, and the NHS Institute for Innovation and Improvement.

Important issues were highlighted at this first meeting of the MDE. These included the need for development of medical directors and their supporting medical management structures in order to better support consultants in handling the impact on service delivery of the reforms (foundation trusts, choice, practice-based commissioning, and payment by results). Since national guidance on New Ways of Working makes it clear that leadership of multidisciplinary teams is not the exclusive right of any one profession, another concern was how to ensure adequate training in team leadership for specialist registrars and consultants. It was proposed that furthe work may be needed to define the indis pensable, non-transferable contributions of psychiatrists to mental health services in order to encourage members of the College to hone their skills in these areas.

The Vice-President and MDE will draw on the intelligence and work of a muchwider network of medical managers in mental health services. Steve Choong, Chairman of the College Management Special Interest Group, will be an ex officio member of the MDE. He will coordinate and transmit advice of the special interest group's wider membership of medical managers through the MDE to the central College Executive Committee (which has replaced the College Council). Anyone interested in joining the special interest group or learning more about its work should contact lan Davidson, Honorary Secretary (email: ian.davidson@cwpnt.nhs.uk). Membership is not restricted to those holding management positions but is open to anyone interested in leadership and management issues in mental health and learning disability services.

In addition, there are flourishing local networks of medical managers working in some College Divisions on how to implement new ways of working. Peter Kennedy would welcome discussion with medical directors/managers who are interested in developing similar local networks where none yet exists (email: peter@kennedy89.freeserve.co.uk)

To launch and shape how this Collegewide network will develop, an overnight stay and day workshop for medical directors is planned for 12 December in York. A larger event for all medical managers and clinical leaders in mental health services is a possibility for next year.

We are currently trying to establish an accurate database of contact addresses for medical directors. We are particularly concerned to include 'heads of psychiatry' in mental health services that do not have a medical director who is a psychiatrist. This is especially likely in Northern Ireland, Scotland, Wales and Ireland. Any medical director or equivalent who has not been contacted directly with details about the workshop in December please send your details to Eva Davison (email: edavison@nyorkdiv.rcpsych.ac.uk).

\section{Building and Sustaining Specialist Child and Adolescent Mental Health Services}

Council Report CR137, June 2006, Royal College of Psychiatrists, $£ 7.50$, $52 \mathrm{pp}$

This document provides guidance to practitioners, managers and commissioners on the capacity and provision of specialist child and adolescent mental health services (CAMHS) in England, Ireland, Northern Ireland, Scotland and Wales. Evidence is collated from a number of sources, including published and unpublished literature and examples of best practice. During consultation the document was shared with practitioners, non-statutory organisations, policy makers and commissioners from the agencies of health, social care, education and justice across the five jurisdictions.

The guidance is designed to be a support for service development that is based on assessment of need. It emphasises that local factors should be taken into account, including deprivation indices, the numbers of Black children and those from minority ethnic groups, and whether the area is rural or urban.

For Tier 2/3 CAMHS, an epidemiologically needs-based service for 0 - to 16-year-olds requires a minimum of 20 whole-time equivalent (wte) clinicians per 100000 total population. Teams must have a range of clinical professionals with cognitive, behavioural, psychodynamic, systemic and medical psychiatric skills. Team capacity should be set at 40 new referrals per wte per year. Clinician keyworker case-load should average at 40 cases per wte across the service, varying according to the type of cases held and the other responsibilities of the clinician. Specialist CAMHS work with Tier 1 professionals is best provided by dedicated primary mental health workers working as a team and closely linked to Tier 2/3 CAMHS. Matching demand and capacity is essential to ensure effective service provision.

Recommendations for the remit and staffing of Tier 4 services are given, including specialist community intensive treatment services, day services and inpatient services. It is recommended that 20-40 in-patient CAMHS beds per 1 million total population are required to provide for children and adolescents up to the age of 18 years with severe mental health problems, and that bed occupancy should be $85 \%$ to ensure availability of emergency beds.

The authors did not find sufficient evidence to provide recommendations for staffing levels for CAMHS for 16 - to 18year-olds, but argue that significant extra resources are needed to extend services to include this age-group. There was a paucity of evidence on infant mental health services and mental health services for children and adolescents with learning disability, substance misuse and forensic problems. However, the mental health needs of these groups must be met and should be provided by specialist CAMHS.

This document is recommended to anyone who is struggling to answer the questions, 'what should specialist CAMHS be doing and how many people do they need to do it?

\section{Role of the consultant psychiatrist in psychotherapy}

Council Report CR139, May 2006, Royal College of Psychiatrists, $f 5.00$, $15 \mathrm{pp}$

This report reviews the range of roles and responsibilities that are undertaken by consultant psychiatrists in psychotherapy. It sets out three core principles.

- Consultant psychiatrists in psychotherapy have a range of roles.

- Consultant psychiatrists in psychotherapy bring to multidisciplinary teams the knowledge, responsibility and ethos associated with the medical profession.

- Consultant psychiatrists in psychotherapy bring specific psychotherapeutic expertise to multidisciplinary teams.

In clinical work these principles mean that consultant psychiatrists in psychotherapy assess and manage complex cases, deal with issues of risk and take special responsibility for patients with a combination of medical and 
psychological issues. Supervision and management of clinical teams are also important clinical tasks.

The important teaching role is discussed in relation to both undergraduate and postgraduate medical education and the education of professions allied to medicine. Strategic advisory and clinical governance responsibilities are discussed and the particular remit to bring a psychologically minded approach to these discussions is highlighted

Finally, the report highlights the future development of the role in relation to the changing role of medical consultants within he health service. It stresses the importance of developing a capacity for flexible ways of working, employing a range of therapeutic modalities, learning new evidence-based therapies and participating in the research base for and development of new treatments. In addition, the changing structure of adult psychiatry is discussed in relation to developing therapeutic roles for consultant psychiatrists in psychotherapy more generally, including involvement in developments such as assertive outreach, crisis intervention and home treatment teams.

\section{reviews}

\section{The Frith Prescribing Guidelines for Adults with Learning Disability}

Sabyasachi Bhaumik \& David Brenford (eds) London: Taylor \& Francis, 2005 f24.95, pp. 155 ISBN 1841845736

As a child it was often said to me that 'Good things come in little parcels'. This sentiment applies to this book, which although being slender contains invaluable information to guide clinicians faced with the task of managing adults with learning disability who have additional mental health problems, behavioural problems and/or epilepsy.

Compared with the general population, individuals with learning disability often respond differently to standard psychiatric (and other) medication and may be exquisitely sensitive to such medication and its side-effects. Many clinicians are justifiably cautious when prescribing for these patients and are often obliged to seek the advice of their more experienced colleagues; advice that may be more anecdotal than evidence based. Thus it was with a sense of professional delight mingled with relief, that I received this book.

The book covers all the major psychiatric disorders and challenging behaviours that most professionals working with people with learning disability are likely to come across in their daily practice. Each chapter has a clear, logical format and benefits from being succinct with a pleasing absence of verbosity. The authors use sub-headings to full effect and bullet points draw attention to important facts. Chapters that are particularly strong are those on the management of epilepsy in people with learning disability and the management of affective disorders.

The authors clarify treatment options / pathways by the liberal use of treatment algorithms in most chapters. Although this strategy is helpful, some of the algorithms are difficult to follow (particularly that for the treatment of aggression (pages 59-61)).

Having read this text several times I am convinced that it will become an invaluable aide, not only to my psychiatric colleagues but also to other mental health professionals and general practitioners, all of whom regularly treat people with learning disability. For the future, the publication of guidelines for prescribing for children and adolescents with learning disability and co-morbid mental health problems, epilepsy and/or challenging behaviours would be very welcome. please?

Angie Stevens Specialist Registrar in Child and Adolescent Psychiatry, Maudsley Hospital, London SE5 8AZ, email: angela.stevens@slam.nhs.uk

\section{Post-Traumatic Stress Disorder:The Management of PTSD in Adults and Children in Primary and Secondary Care}

National Collaborating Centre for Mental Health London \& Leicester: Gaskell \& The British Psychological Society, 2005, f50.00, pp.168 ISBN: 190467125

Post-traumatic stress disorder (PTSD) has its believers and non-believers, but the balance appears to be moving in favour of the former. Despite numerous descriptions of the disorder since the First World War (and before), it was not a formally recognised clinical diagnosis until fairly recently.

The increased number of victims of violence within our society, including political refugees and the victims of the recent bomb attacks in London, places PTSD at the centre of the current health and political agendas. All of this makes this book more than welcome, as it responds to the clear need for understanding, training and clinical guidelines.

This book introduces the reader to the concept of PTSD, mainly from the medical/clinical point of view and includes some observations about the psychosocial dimensions. We are offered a summary of the majority of well-conducted randomised clinical trials of its treatment modalities, both psychotherapeutic and pharmacological, both in adults and children, in whom its presentation is less well described. It covers disaster planning (very topical) and early intervention, and makes recommendations for future research. Furthermore, there is a very moving and enlightening chapter dedicated to the views and experiences of sufferers and carers from different backgrounds.

It is important to note, however, that anyone looking to gain a thorough understanding of more complex and severe cases of PTSD will not find it here. The main research trials select populations of the more simple cases of trauma - this might be owing to the costs, length and complexity of including studies of the more complicated and severe clinical cases. This book also misses the opportunity to satisfy the reader's curiosity in relation to newer treatments for PTSD such as eye movement desensitisation and reprocessing (EMDR), which is briefly described but only from the perspective of cognitive-behavioural therapy, which predominates in this book.

As a summary of current trends and practices, however, this book is invaluable. It will be useful to a range of health and non-health workers, including general practitioners, psychiatric services, children's services, psychotherapists, and others within the National Health Service and non-statutory services.

Nuri Gene-Cos Traumatic Stress Service, Clinical Treatment Centre, Maudsley Hospital, London SE5 8AZ, email: nurigenecos@slam.nhs.uk

\section{Reducing the Stigma of Mental Illness: A Report from a Global Programme of the World Psychiatric Association}

Norman Sartorius \& Hugh Schulze Cambridge: Cambridge University Press, 2005, f30.00, 233 pp. ISBN: 0-521-5493-4

Essentially a factual report upon an international programme to reduce stigma, this book develops many interesting ideas beyond those which might be expected from the title. It gives a detailed account 